\title{
A Comprehensive Review on Dental Calculus
}

${ }^{1}$ Suchetha Aghanashini, ${ }^{2}$ Bhavana Puvvalla, ${ }^{3}$ Darshan B Mundinamane, ${ }^{4} \mathrm{SM}$ Apoorva, ${ }^{5}$ Divya Bhat, ${ }^{6}$ Manjari Lalwani

\begin{abstract}
Dental calculus is a hard deposit that is formed by calcification of dental plaque primarily composed of calcium phosphate mineral salts which is deposited on natural teeth and restorations and is covered by a layer of unmineralized plaque. These hard deposits may form coronal to or apical to the gingival margin, hence named accordingly as supragingival and subgingival calculus respectively. The distribution of calculus is very versatile and it differs from individual to individual, from tooth to tooth, and from surface to surface. So, a thorough knowledge on prevalence of calculus is important for the clinician in outlining the treatment plan. It is a well-known fact that calculus is itself not an inducing agent for pathological changes that occur in gingival tissues; instead it is covered by a layer of unmineralized plaque which is proven to be the key etiological agent involved in these pathogenic mechanisms. But, attributing to the porosity of calculus and its ability to retain bacterial antigens makes it an important contributing factor in initiating and accentuating periodontal disease progression. In this review, we made an attempt to discuss various aspects of calculus composition, its formation, and its etiological significance in periodontal disease progression.
\end{abstract}

Keywords: Calculus, Gingival margin, Periodontal diseases, Prevalence, Unmineralized plaque.

How to cite this article: Aghanashini S, Puvvalla B, Mundinamane DB, Apoorva SM, Bhat D, Lalwani M. A Comprehensive Review on Dental Calculus. J Health Sci Res 2016;7(2):42-50.

\section{Source of support: Nil \\ Conflict of interest: None}

\section{INTRODUCTION}

Periodontitis is a chronic inflammatory disease of supporting tissues of tooth which is caused by specific microorganisms in a susceptible host. ${ }^{1}$ Bacterial plaque and calculus are considered as major etiological agents in the initiation and progression of periodontal diseases. ${ }^{2}$ Calculus is defined as a hard deposit that is formed by mineralization of dental plaque on the surfaces of natural teeth and dental prosthesis, generally covered by a layer of unmineralized plaque. ${ }^{3}$ Calculus is derived from Greek words Calcis-lime stone, Tartar-white encrustation inside

\footnotetext{
${ }^{1}$ Professor and Head, ${ }^{2,6}$ Postgraduate Student, ${ }^{3,4}$ Reader ${ }^{5}$ Senior Lecturer

${ }^{1-6}$ Department of Periodontology, DA Pandu Memorial RV Dental College and Hospital, Bengaluru, Karnataka, India
}

Corresponding Author: Bhavana Puvvalla, Postgraduate Student, Department of Periodontology, DA Pandu Memorial RV Dental College and Hospital, Bengaluru, Karnataka, India Phone: +917411244744 e-mail: Dr.puvvalla@gmail.com casks and also called as Tartar, Disambiguation, Calcis, Odontolithiasis and Fossilized plaque.

\section{CASE REPORT}

- Hippocrates (460-377 BC) was the foremost person whose writings showed a relation between dental deposits and oral diseases. He noted the harmful effects of calculus (pituita) on gums and teeth.

- Albucasis (936-1013) evidently illustrated the association between calculus and disease and advocated thorough removal of deposits.

- Paracelsus a Swiss/German physician and alchemist introduced the term tartar as a description for a variety of stony concretions that form in humans.

- For nearly 5,000 years, calculus was considered to be the prime etiologic agent in periodontal disease. In the past 25 years, however, calculus has been overthrown by plaque, and the hardened criminal has come to be viewed as a fossilized remnant of minor significance. This shift in perception became mainly apparent in the 1960s and was largely a response to two lines of investigation.

1. Reports on experimental and electron microscopic studies of developing plaque and calculus confirmed that supra- and subgingival calculus were mineralized plaque covered by a layer of unmineralized bacteria

2. The experimental demonstration in humans that plaque permitted to accumulate in the absence of oral hygiene results in a gingivitis, which is reversible on the resumption of tooth cleaning.

\section{CLASSIFICATION}

- According to location

- Supragingival calculus

- Subgingival calculus

- According to source of mineralization

- Salivary calculus

- Serumal calculus (Jenkins, Stewart 1966)

- According to surface

- Exogenous

- Endogenous (Melz 1950)

- According to initiation and rate of accumulation, calculus formers are classified as:

- Noncalculus formers

- Slight calculus formers

- Moderate calculus formers

- Heavy calculus formers. ${ }^{4}$ 
A Comprehensive Review on Dental Calculus

\begin{tabular}{|c|c|c|c|}
\hline SI. no. & Feature & Supragingival calculus & Subgingival calculus \\
\hline 1 & Defined as & $\begin{array}{l}\text { Tightly adhering calculus deposit that forms on the } \\
\text { crowns of the teeth coronal to the gingival margin }\end{array}$ & $\begin{array}{l}\text { Calcified deposit that forms on the tooth surface } \\
\text { below the free margin of gingiva }\end{array}$ \\
\hline 2 & Location & Forms coronal to the gingival margin & Deposits present apical to the crest of marginal gingiva \\
\hline 3 & Source & Derived from the salivary secretions - salivary calculus & Derived from the gingival exudate - seruminal calculus \\
\hline 4 & Distribution & $\begin{array}{l}\text { Symmetrical arrangement on teeth, more on facial } \\
\text { surfaces of maxillary molars and lingual surfaces of } \\
\text { mandibular anterior teeth }\end{array}$ & $\begin{array}{l}\text { Related to pocket depth, heavier on proximal } \\
\text { surfaces }\end{array}$ \\
\hline 5 & Color & It is white, yellow in color & Brown/greenish black in color \\
\hline 6 & Consistency & Hard and clay like & Hard and firm/flint or glass like \\
\hline 7 & Composition & $\begin{array}{l}\text { More brushite and octa calcium phosphate } \\
\text { Less magnesium whitelockite }\end{array}$ & $\begin{array}{l}\text { Less brushite and octa calcium phosphate. More } \\
\text { magnesium whitelockite }\end{array}$ \\
\hline \multirow[t]{2}{*}{8} & Other contents & Sodium content is less & Sodium content increases with the depth of the pocket \\
\hline & & Salivary proteins are present & Salivary proteins are absent \\
\hline 9 & Visibility & Clinically visible & Not visible on routine clinical examination \\
\hline 10 & Attachment & Easily detached from the tooth & Firmly attached to the tooth surface \\
\hline
\end{tabular}

\section{COMPOSITION OF CALCULUS}

Calculus consists of both inorganic and organic components.

\section{Inorganic Contents (70-90\%)}

Principle elements:

- Calcium-39\%

- Phosphorous $-19 \%$

- Carbon dioxide $-1.9 \%$

- Magnesium $-0.8 \%$

- Trace amounts of $\mathrm{Na}, \mathrm{Ba}, \mathrm{Zn}, \mathrm{Str}, \mathrm{Br}, \mathrm{Cu}, \mathrm{Ag}, \mathrm{Al}, \mathrm{Fe}, \mathrm{Fl}$ Components:

- Calcium phosphate $-75.9 \%$

- Calcium carbonate $-3.1 \%$

- Magnesium phosphate - traces and other metals Crystal forms:

- Hydroxyapatite (HA) - 58\%

- Octa calcium phosphate (OCP) $-21 \%$

- Magnesium whitelockite (MWL) - $12 \%$

- Brushite (BS) $-9 \%$

Supragingival calculus - HA and OCP are detected most frequently.

Subgingival calculus - MWL is present in high concentrations and same HA content.

Mandibular anterior region - BS is more common.

Posterior areas - MWL is more common (Table 1). ${ }^{6}$

\section{PREVALENCE}

An emphasis on the prevalence of calculus is important as it not only provides an outlook of health but also helps us in plotting the treatment required and professional services accordingly. There are a multitude of recent reports available on prevalence of calculus from all over the world. Several studies assessed calculus applying the CPITN index for estimating the population need for periodontal care. There are also additional studies that helped us in defining the prevalence of calculus. ${ }^{7}$ Among ${ }^{8}$ various studies, a study done by Anerud et al in 1991 for a 15-year period observed the periodontal status of two groups: (1) Sri Lankan tea laborers group and (2) Norwegian academicians group. Ready access to preventive dental care is available for Norwegian group in contrary to Sri Lankan individuals. They noticed that calculus accumulation was symmetrical and was first accumulated on facial surface of maxillary molars and lingual surfaces of mandibular incisors. With the increase in age, the deposition of supragingival calculus continues reaching to a maximal calculus score around 25 to 30 years of age, and by age 45 , almost all the teeth would have been covered by calculus except premolars in some instances. Norwegians enjoyed the privileged dental services and showed approximately $70 \%$ of interproximal calculus free sites by the age of 40 to 50 years. Hence, concluding that individuals who did not practice good oral hygiene are at a higher risk of attachment loss and ultimately tooth loss when compared to teeth without calculus.

More recently, NHANES III survey evaluated 9,689 adults in the United States between 1988 and 1994 and observed that $91.8 \%$ of the subjects had noticeable calculus and $55.1 \%$ had subgingival calculus. ${ }^{9}$

\section{FORMATION OF CALCULUS}

After the tooth eruption or a dental prophylaxis, pellicle proteins rapidly adsorb onto the enamel surface which favors bacterial adhesion and subsequent development of biofilm occurs. Maturation of biofilm proceeds with 
the characteristic microbial maturation of initial Grampositive coccoidal organisms followed by outgrowth of filamentous bacteria leading to the development of plaque. Mineralizing agents from saliva and GCF for supra- and subgingival calculus respectively enter the biofilm leading to mineralization of plaque intercellular matrix. Hereafter, calcium ions from saliva are removed by chelation promoting binding of calcium with carbohydrate/protein complexes leading to the precipitation of crystalline calcium phosphate salts, and coalescence of these crystals aids in the formation of calcified mass, thereby leading to calculus formation. ${ }^{5}$

\section{THEORIES OF CALCULUS FORMATION}

Many theories were proposed to better understand the mechanism of calculus formation. They are as follows:

- Booster mechanism

- Epitactic concept

- Inhibition theory

- Transformation theory

- Bacterial theory

- Enzymatic theory

\section{Booster Mechanism}

\section{Mechanism 1}

Major salivary ducts secrete saliva at a high $\mathrm{CO}_{2}$ tension, about 54 to $65 \mathrm{~mm} \mathrm{Hg}$; but the pressure of $\mathrm{CO}_{2}$ in atmospheric air is only about $0.3 \mathrm{~mm} \mathrm{Hg}$. As a result of large disparity in $\mathrm{CO}_{2}$ tension, saliva emerging from the salivary ducts loses $\mathrm{CO}_{2}$ to the atmosphere. $\mathrm{pH}$ in saliva will increase when $\mathrm{CO}_{2}$ escapes since $\mathrm{pH}$ in saliva depends largely on the ratio between bicarbonates and free carbonic acid. Phosphoric acid dissociation increases with rise in the alkalinity, thus increasing the concentration of less soluble secondary and tertiary phosphate ions. This boost in phosphate ions concentration leads to a situation where solubility product of calcium phosphate is exceeded and crystals form (Flow Chart 1).

Flow Chart 1: Booster mechanism
Atmosphere $\mathrm{CO}_{2}$
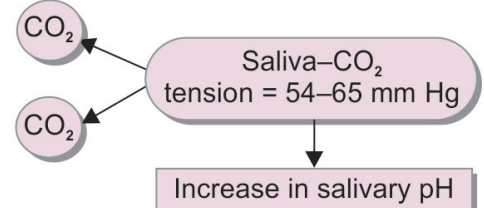

Increase in salivary $\mathrm{pH}$

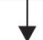

Dissociation of tension $=0.3 \mathrm{~mm} \mathrm{Hg}$ phosphoric acid

Phosphate ions

\section{Mechanism 2}

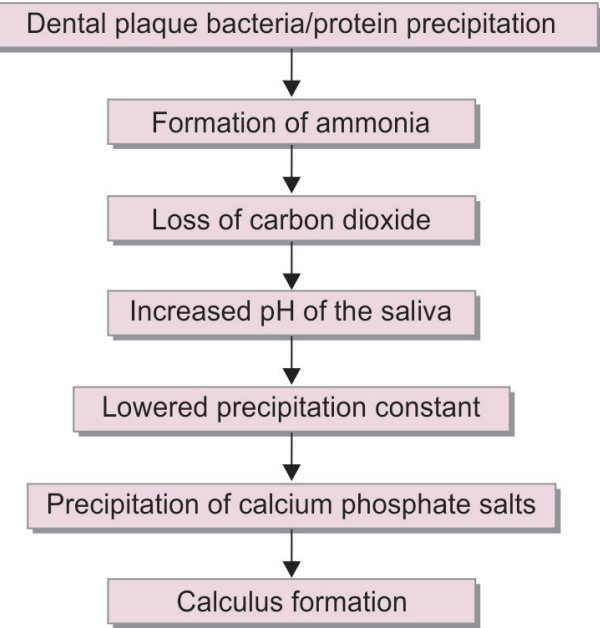

\section{Epitactic Theory}

In saliva the concentration of certain ions like calcium and phosphate is not high enough to precipitate but is ample enough to promote the growth of hydroxyapatite crystals once an initial seed or nucleus is formed. The term epitactic refers to crystal formation through seeding by another compound which is similar to hydroxyapatite crystals, leading to precipitation of calcium salts from the metastable solution of saliva. Seeding agents provoke small foci of calcification enlarge and coalesce to form the calcified mass. Intercellular matrix or plaque plays an important role. Calcification will be initiated by a carbohydrate/protein complex which removes calcium from saliva by chelation process and binds with the nuclei that stimulates subsequent deposition of minerals.

\section{Inhibition Theory}

This theory assumes about calcification as occurring only at specific sites because of existence of an inhibiting mechanism at noncalcifying sites. According to this theory, the sites where calcification occurs, the inhibitor is apparently altered or removed. Pyrophosphate is thought to be one possible inhibiting substance and other possible inhibiting substances include polyphosphates. Alkaline pyrophosphatase is the enzyme involved in controlling mechanism which hydrolyzes the pyrophosphate to phosphate (Russell and Fleisch 1970) and this pyrophosphate prevents the initial nucleus from growing and inhibits their calcification possibly by poisoning the growth centers of the crystals.

\section{Transformation Theory}

Most noticeable hypothesis states that hydroxyapatite need not arise exclusively via epitaxis or nucleation. Octa calcium phosphate is formed by the transformation of 
amorphous noncrystalline deposits and brushite and then transformed to hydroxyapatite (Eanes et al 1970). It has been suggested that controlling mechanism in transformation mechanism can be pyrophosphate (Fleisch et al 1968).

\section{Bacteriological Theory}

According to this theory, the primary cause of calculus formation is oral microorganisms and their involvement in attachment to the tooth surface. Leptotrichia and actinomyces have been considered most often as the causative microorganisms.

\section{Enzymatic Theory}

According to this theory, calculus formation is the resultant of the action of phosphatases derived from either oral tissues or oral microorganism on some salivary phosphate containing complex, most probably phospheric esters of the hexophosphoric group. ${ }^{5}$

\section{CALCULUS ATTACHMENT}

The following four modes of attachment have been described

1. Attachment by means of organic pellicle on enamel

2. Mechanical interlocking in cemental resorption lacunae

3. Close adaptation of calculus undersurface depressions to gently sloping mounds on the unaltered cementum surface

4. Penetration of calculus bacteria in cementum. But this mode of attachment was not acknowledged. ${ }^{6}$

\section{ATTACHMENT OF CALCULUS ON IMPLANT}

- Calculus attachment to pure titanium is less intimate than to root surfaces structure.

- Smooth machined implants have less micro porosities for retention. This would mean that calculus may be chipped off from implants without affecting it. ${ }^{10}$

\section{MICROBIOLOGY OF DENTAL CALCULUS}

The average microscopic count of bacteria in unmineralized dental plaque has been calculated to be up to $2.1 \times 10 \mathrm{mg}$ wet weight. Lactate dehydrogenase and alkaline and acid phosphatase activities have been identified in dental plaque suggestive of a boosted calcification by the plaque enzymes. In supragingival calculus, viable aerobic and anaerobic bacteria have been detected while subgingival calculus provides an excellent environment for further microbial adhesion and growth. Periopathogens, such as Aggregatibacter actinomycetemcomitans, Porphyromonas gingivalis, and Treponema denticola have been found within the lacunae of both supra- and subgingival calculus. Bacteria are not essential for calculus formation, but they enable its development. Hence, high amount of calculus indicates that oral hygiene has been poor for months or even years. ${ }^{6}$

\section{ETIOLOGICAL SIGNIFICANCE OF CALCULUS IN PERIODONTAL DISEASE}

It is a quite challenging task to distinguish the effects of calculus and plaque on gingiva, because the former is always covered by a layer of plaque. There is always a correlation between the presence of calculus and prevalence of gingivitis though greater association was found between plaque and gingivitis. This association may lead to initiation and progression of periodontal diseases.

The rough calculus surface may not, in itself, induce inflammation in the adjacent periodontal tissues, instead it serves as an ideal substrate for subgingival microbial colonization ${ }^{11}$ and also

- acts as a niche which harbors bacterial plaque

- acts as an irritant to the periodontal tissues

- distends the periodontal pocket wall

- inhibits the ingress of polymorphonuclear leukocytes. So, regardless of its primary or secondary relationship in pocket formation, i.e., periodontal disease progression and although the principal irritating features is its surface plaque rather than its interior, calculus is a significant pathogenic factor in periodontal disease. ${ }^{6}$

But, various studies have emphasized on the aspect that whether sterilized section of calculus has some role in periodontal disease progression. Among various studies, a study conducted by Don Allen and Kerr (1965) tested the sterility of calculus and its reaction and compared with unsterile calculus in guinea pigs histologically. A portion of calculus obtained from periodontitis patients and the calculus is autoclaved to achieve sterilization and then put in a hotair oven to evaporate the moisture which had accumulated in them as a result of autoclaving. This portion of sterilized calculus in injected into the intraperitoneal tissues of a group of guinea pigs and the other group received an injection of unsterilized calculus. Results showed that the response sterile human calculus is a granulomatous, foreign body reaction whereas to unsterile human calculus is a suppurative reaction with a tendency for abscess formation concluding that sterile calculus is a mild irritant and has no etiological significance when compared to calculus with microorganisms. ${ }^{12}$

In dental research, the association between dental calculus with periodontal health has doubtlessly been one of the most studied topics. For several millennia past to 1960 , dental calculus was considered to be a primary 
etiologic factor in the initiation and progression of periodontal diseases.

In the decade of 1970s, the enhanced understanding of the microbiological contribution to periodontal disease decline interest in calculus as a specific etiologic agent. The last decade has seen renewed interest in supra- and subgingival calculus effects on disease processes, on the one hand, due to the commercial success of toothpastes sold for the control of supragingival calculus, and on the other hand, due to the success of phase I periodontal therapy (scaling and root planing, with subgingival calculus debridement) in treating early periodontal disease as documented by TEN CATE and MANDEL and GAFFEN ${ }^{13}$

Ainamo (1970) found a high positive correlation between calculus (both supra- and subgingival and gingivitis) in 154 army recruits between the ages of 19 and 22. He employed the retention index (RI) which discriminates between plaque associated with calculus and plaque associated with caries and noted a positive correlation between the RI and gingivitis. A higher correlation was noted between gingivitis and calculusrelated plaque than with cariogenic plaque. Ainamo ${ }^{14}$ also spotted attention to his finding that there was increased gingivitis as well as calculus deposits on oral than on facial surfaces of lower second premolars and first and second molars. One possible justification is that this is the area where the salivary influence is greatest, hence, supragingival calculus is most abundant and suggests that the pathogenicity of calculus plus overlying plaque may be greater than that of plaque alone.

Alexander (1971) observed the regional distribution of bacterial plaque, supra- and subgingival calculus, and gingival inflammation in 200 dental students and 200 patients visiting a dental clinic. He noticed that the prevalence of gingival inflammation is greatly exhibited in the papillary areas and the buccal margins the lowest which coincides with the greatest prevalence of subgingival calculus on the interproximal surface and the buccal margins the lowest, concluding that the surfaces with calculus exhibited more gingivitis than the surfaces with plaque alone. ${ }^{15}$

Buckley ${ }^{16}$ examined the prevalence of subgingival and supragingival calculus among 300 teenagers, aged 15 to 17 , evenly distributed by age and sex. He found greater prevalence of subgingival calculus when compared to supragingival but showed the same distribution pattern. A strong correlation was found between the buccal and lingual gingival indices and their respective plaque and supra- and subgingival calculus indices. Pearson correlation analysis indicated a higher degree of correlation for gingival indices vs plaque than for gingival indices vs calculus and a higher degree of correlation for subgingival than for supragingival calculus.

Furthermore, Lennon and Clerehugh ${ }^{17}$ in a 2 -year longitudinal study elucidated the role of subgingival calculus in periodontal disease in teenagers. This investigation included 229 children in the age group of 14 to 16 and the authors concluded that the presence of subgingival calculus was the best predictor of future attachment loss.

Axelsson and Lindhe ${ }^{22}$ during a 6-year longitudinal study in 555 adults observed that whether occurrence of caries and periodontal disease progression can be prevented by maintaining oral hygiene and repeated prophylaxis. Each prophylactic session is given every 2 months during the first 2 years, and every 3 months thereafter and included a complete scaling and root planing, combined with oral hygiene instructions. The study concluded that subjects who utilized proper oral hygiene techniques had negligible signs of gingivitis and periodontal tissue attachment loss and developed no new carious lesions. Similar strategies of frequent recalls are characteristic of all the adult plaque control studies at Goteborg. ${ }^{18-21}$ All these studies highlighted that the plaque control and professional oral prophylaxis had certainly played an important role in maintaining the gingival and periodontal health.

Tagge et $\mathrm{al}^{23}$ in 22 patients assessed clinically and microscopically, the soft tissue response in suprabony periodontal pockets after treatment by root planing and oral hygiene $v s$ oral hygiene measures alone. All the therapies decreased the incidence and severity of gingivitis along with pocket depth. However, root planing combined with oral hygiene measures resulted in a statistically significant improvement when compared to personal oral hygiene measures alone. This is because the tooth brushing limited its effectiveness by the presence of subgingival deposits on the nonroot-planed surfaces that resulted in no significant pocket reduction and gain in attachment levels than in those treated by root planing with oral hygiene prophylaxis.

Hellden et $\mathrm{al}^{24}$ studied advanced periodontal disease in 12 patients. The presence of plaque, gingival inflammation, probing depths, and attachment levels was assessed for all teeth in 12 patients with chronic, advanced periodontitis. After the initial examination, patients were given detailed oral hygiene instructions and divided into four groups.

Group 1: No treatment,

Group 2: Scaling and root planing alone,

Group 3: Administration of tetracycline alone, and scaling and

Group 4: Root planing combined with the administration of tetracycline. 
Following initial therapy all patients were reexamined at 8 and 25 weeks. Both plaque index (PI) and gingival index (GI) scores were recorded which decreased significantly in all groups. In group 2 the GI scores were significantly lowered and also there was significant reduction in probing depth. It was also observed that there was a trend to gain of attachment in the treated areas, suggesting that removal of calculus led to improvement in gingival health. ${ }^{24}$

Morrison et $\mathrm{al}^{25}$ in their 90 subjects examined the effects of initial, nonsurgical, periodontal treatment (the hygienic phase) on the clinical severity of periodontitis in pockets varying from 1 to $>7 \mathrm{~mm}$. The results showed that there is a significant reduction in inflammation following removal of the plaque and calculus deposits and the improvement was great enough to call for reassessment of the need for surgery in some instances. In evaluating the various factors involved in the hygienic phase, it was eminent that the changes in plaque scores could not be correlated with attachment level gain and pocket depth reduction and the authors considered a very important to the success of the hygienic process is the significant reduction in subgingival calculus.

Chawla et $\mathrm{al}^{26}$ investigated the effect of various dental prophylaxis regimens in 1,605 subjects between the age group of 12 and 26 over a period of 2 years. The results showed that scaling along with oral hygiene instructions at 6-month intervals provided the maximum benefit. Concluding, that the removal of bacterial plaque alone did not show significant improvement in periodontal health but the removal of calculus was directly correlated with the improvement in periodontal health. They also spotted out that this did not mean that regular oral hygiene measures are not important, but rather that "viable bacterial plaque, retained on and around the retention areas provided by calculus, unless removed, may not obviously be as effective."

A morphologic study by Jones ${ }^{27}$ and Bauhammers et $\mathrm{al}^{28}$ in their scanning electron microscopic (SEM) studies observed the marked roughness of the outer surface of calculus leading to retention of bacterial plaque.

A comparative study done by Friskopp and Hammarstrom ${ }^{29}$ used SEM in their study of supra- and subgingival calculus. The morphology of supra- and subgingival calculus on extracted teeth was studied using SEM. The differences were observed in the nature of microbial coverings. Supragingival calculus is dominated by filamentous organisms, oriented at right angles to the surface whereas subgingival calculus was covered by cocci, rods, and filaments with no distinct pattern of orientation. When sodium hypochlorite was used in some of the specimens they lost their soft covering and channels of the same dimension as the filamentous organisms were found penetrating into the calculus.

Friskopp ${ }^{30}$ in a further study also noted cavities of noncalcified material of the ultrastructure of supragingival calculus, and subgingival deposits tended to be more homogeneous.

Shirato et $\mathrm{al}^{31}$ reported the presence of tubular holes in calculus. These holes appeared to be areas of uncalcified bacteria which is surrounded by calcified matrix. There were also areas where the bacteria were calcified but were surrounded by a noncalcified space. All of the morphologic studies attest to the porous nature of the calculus deposits.

Patters et $\mathrm{al}^{32}$ assayed the bone resorbing activity (using an organ culture system) and the presence of antigens of Bacteroides gingivalis in plaque, calculus, cementum, and dentin obtained from roots of extracted teeth from patients with severe periodontitis. Significant stimulation of bone resorption was found in the preparations from periodontally involved cementum and in all samples of calculus. The levels of bone resorbing activity were higher. This study provides the strongest evidence to date of the pathogenic potential of subgingival calculus. ${ }^{32}$

An experimental study to know the permeability of human and rat dental calculus is done by Baumhammers et $\mathrm{al}^{28}$ in which they used a series of dyes, titrated endotoxin, and titrated glycine. The results showed that the human calculus was partially permeated in one hour and completely permeated by the dyes in 24 hours. Radioautographs showed progressive penetration of the titrated glycine and endotoxin with time. This led to hypothesis that dental calculus can act as a reservoir for irritating substances from microbial plaque and tissue lysis.

\section{INDICES USED FOR CALCULUS DETECTION ${ }^{33}$}

\section{Oral Calculus Index $(\mathrm{OCI})$ (Greene and Vermilion, 1964)}

It is the component of the oral hygiene index. An explorer is used to estimate the surface area covered by supragingival calculus and to probe for the subgingival calculus. ${ }^{33}$ Scores are assigned according to the following criteria:

- No calculus

- Supragingival calculus covering more than one-third of the exposed tooth surface

- Supragingival calculus covering more than one-third but not more than two-thirds of tooth surface

- Supragingival calculus covering more than one-third but not more than two-thirds of tooth surface and/or a continuous band of subgingival calculus.

After the scores for debris and calculus are recorded, the index values are calculated. For each individual, the 
debris scores are totaled and divided by the number of surfaces scored.

\section{Calculus Index - Cl (Ramfjord, 1959)}

The scores on calculus for each individual tooth examined are added and the sum divided by the number of the teeth examined to yield the index on calculus. The following teeth were selected as indicators 16, 21, 24, 36, 41, and 44 . Calculus recorded as follows:

- No calculus

- Supragingival calculus extending only slightly below the free gingival margin (not more than $1 \mathrm{~mm}$ )

- Supragingival calculus covering more than one-third but not more than two-thirds of tooth surfaces

- Supragingival calculus covering more than two-thirds of exposed tooth surfaces.

\section{Calculus Surface Severity Index (CSI) (Ennener et al, 1961)}

The CSI assesses the presence or absence of calculus on the four surfaces of the four mandibular incisors. Each surface is given a score of 1 for the presence of calculus or 0 for the absence of calculus. Maximum score for each subject is 16. In applying the scoring method, calculus was considered to be present in any amount, supragingival or subgingival, and it could be detected either visually or by touch. If the examiner was uncertain about the presence of calculus on a given surface, the surface was called calculus free.

\section{Calculus Rating (Volpe and Manhold, 1962)}

Calculus formation in vivo is performed using a colored periodontal probe placed against the lingual surface of the anterior tooth that will be scored with the probe and placed at the most inferior border of any calculus present. When the different colors at the probe end represent units, the amount of calculus present can be measured:

- U - No calculus

- U-1 mm of calculus

- U-2 mm of calculus

- U-3 mm of calculus

- $\mathrm{U}-4 \mathrm{~mm}$ of calculus

\section{Marginal Line Calculus Index (MLC-I) (Muhlanann and Villa, 1967)}

- No calculus

- Calculus observable, but less than $0.5 \mathrm{~mm}$ in width and/or thickness

- Calculus not exceeding $1 \mathrm{~mm}$ in width and/or thickness

- Calculus exceeding $1 \mathrm{~mm}$ in width and/or thickness.

\section{CALCULUS DETECTION}

- Visual examination

- Gentle air blast

- Transillumination

- Gingival tissue color change

- Tactile examination

- Probe

- Explorer

- Radiographs

\section{Visual Examination}

Good lighting helps us to easily visualize supra- and subgingival calculus just below the gingival margin. When light deposits of supragingival calculus are wet with saliva they are frequently difficult to visualize.

Supragingival calculus can be dried using compressed air until it is readily visible and chalky white. Air may also be directed into the pocket in steady stream to visualize the subgingival deposits by deflection of gingival margin away from the tooth surface.

\section{Tactile Exploration}

Requires the skilled use of fine pointed explorer or probe. The explorer is held with light but stable modified pen grasp. The pads of the thumb and the middle finger should perceive the slight vibration conducted through the shank.

Fine-pointed explorer or probe is used for tactile sensation and is held with light but stable modified pen grasp. Slight vibrations are perceived by pads of the thumb and the middle fingers through the shank.

Method: First, a stable finger rest is established and then the instrument tip is inserted to the pocket depth. In a vertical direction light exploratory strokes are activated. On contact with the calculus, the tip of probe is advanced more apically till the termination of calculus is felt on root surface. Generally, 0.2 to $1.0 \mathrm{~mm}$ is the distance appreciated between apical edge of calculus and bottom of the pocket. Proximal surfaces when explored with an instrument tip, it should be extended at least halfway across the surface past the contact area.

\section{Radiographs}

Interproximal calculus, a highly calcified deposit, can readily be detected as radiopaque projections protruding into the interdental space. The apical location of plaque is not sufficiently calcified to be visible on radiograph, so the calculus location does not indicate bottom of periodontal pocket. Hence, conventional oral radiography was a poor diagnostic method for the detection of calculus (Buchanan et al, 1987). 
ADVANCE DIAGNOSTIC AIDS

\section{Calculus Detection Systems Only}

PERIOSCOPE-Fiberoptic endoscopy-based technology $y^{34,35}$ DETECTAR - Spectro-optical technology

DIAGNODENT - Autofluorescence-based technology

\section{Calculus Detection + Removal Systems}

PERIOSCAN - Ultrasound technology

KEYLASER - Laser-based technology

\section{CONCLUSION}

While the bacterial plaque that coats the teeth is the chief causative factor in the initiation and progression of periodontal disease, the removal of subgingival plaque and calculus constitutes the foundation stone of periodontal therapy. Calculus plays a key role in maintaining and accentuating periodontal disease by withholding the plaque in close contact with the tooth surface and gingival tissue, leading to various pathological changes thereby creating areas where plaque removal is impossible. Therefore, adequate skill of the clinician is essential to remove the calculus and other irritants, which forms the basis for adequate periodontal and prophylactic therapy.

\section{REFERENCES}

1. Meghana DP, Quaid JS, Arvind S. Interrelationship between chronic periodontitis and anemia: A 6-month follow-up study. J Periodontol 2014 Mar 6;18(1):19-25.

2. Masud M, Zahari HIM, Sameon ASN, Mohamed NAH. Manual and electronic detection of subgingival calculus: Reliability and accuracy. Int J Adv Med Res (JAMR) 2014 May;1(1):52-56.

3. Carranza's clinical periodontology. 11th ed. Chapter 22. p. 291.

4. Tannenbaum PJ, Posner AS, Mandel ID. Formation of calcium phosphates in saliva and dental plaque. J Dent Res 1976 Nov-Dec;55(6):997-1000.

5. Sahithya, RS. Essentials of periodontology. 1st ed. Chapter 20: Dental calculus. p. 213-216.

6. Carranza's clinical periodontology. 10th ed. Chapter 10. p. 172-173.

7. White DJ. Dented calculus: Recent insights into occurrence, formation, prevention, removal and oral health effects of supragingival and subgingival deposits. Eur J Oral Sci 1997 Oct;105(5 Pt 2):508-522.

8. Anerud A, Loe H, Boysen $\mathrm{H}$. The natural history and clinical course of calculus formation in man. J Clin Periodontol 1991 Mar;18(3):160-170.

9. Carranza's clinical periodontology. 9th ed. Chapter 11. p. 183.

10. Matarasso S, Quaremb G, Corraggio F, Vaia E, Cafiero C, Lang NP. Maintenance of implants: An in vitro study of titanium implant surface modifications subsequent to the application of different prophylaxis procedures. Clin Oral Implants Res 1996 Mar;7(1):64-72.

11. Jepsen S, Deschner J, Braun A, Schwarz F, Eberhard J. Calculus removal and the prevention of its formation. Periodontol 2000 2011 Feb;55(1):167-188.

12. Allen DL, Kerr DA. Tissue response in the guinea pig to sterile and non-sterile calculus. J Periodontol 1965 Mar-Apr;36: 121-126.

13. Mandei ID, Gaffar A. Calculus revisited: A review. J Clin Periodontal 1986 Apr;13(4):249-257.

14. Ainamo J. Concomitant periodontal disease and dental caries in young adult males. Suom Hammaslaak Toim 1970;66(6):301-364.

15. Alexander AG. A study of the distribution of supra and subgingival calculus, bacterial plaque and gingival inflammation in the mouths of 400 individuals. J Periodontol 1971 Jan;42(1):21-28.

16. Buckley LA. The relationships between irregular teeth, plaque, calculus and gingival disease. Br Dent J 1980 Feb; 148(3):67-69.

17. Lennnon, MA.; Clerehugh, V. The identification of teenage children at high risk of periodontal disease. Periodontal disease in Western Europe. Chicago: Quintessence; 1984. p. 211-216.

18. Nyman S, Rosling B, Lindhe J. Effect of professional tooth cleaning on healing after periodontal surgery. J Clin Periodontol 1975 Apr;2(2):80-86.

19. Nyman S, Lindhe J. Considerations in the treatment of patients with multiple teeth with furcation involvements. J Clin Periodontol 1976 Feb;3(1):4-13.

20. Rosling B, Nyman S, Lindhe J. The effect of systematic plaque control on bone regeneration in infrabony pockets. J Clin Periodontol 1976 Feb;3(1):38-53.

21. Axelsson P, Lindhe J. Effect of controlled oral hygiene procedures on caries and periodontal disease in adults. Results after 6 years. J Clin Periodontol 1981 Jun;8(3):239-248.

22. Axelsson P, Lindhe J. Effect of controlled oral hygiene procedures on caries and periodontal disease in adults. J Clin Periodontol 1978 May;5(2):133-151.

23. Tagge DL, O'Leary TJ, El-Kafrawy AH. The clinical and histological response of periodontal pockets to root planning and oral hygiene. J Periodontol 1975 Sep;46(9):527-533.

24. Hellden LB, Listgarten MA, Lindhe J. The effect of tetracycline and/or scaling on human periodontal disease. J Clin Periodontol 1979 Aug;6(4):222-230.

25. Morrison EC, Ramfjord SP, Hill RW. Short-term effects of initial, nonsurgical periodontal treatment (hygienic phase). J Clin Periodontol 1980 Jun;7(3):199-211.

26. Chawla TN, Nanda RS, Kapoor KK. Dental prophylaxis procedures in control of periodontal disease in Lucknow (rural) India. J Periodontol 1975 Aug;46(8):498-503.

27. Jones SJ. Calculus on human teeth. Apex 1972;6:55-59.

28. Baumhammers A, Conway JC, Saltzberg D, Matta RK. Scanning electron microscopy of supragingival calculus. J Periodontol 1973 Feb;44(2):92-95.

29. Friskopp J, Hammarstrom L. A comparative, scanning electron microscopic study of supragingival and subgingival calculus. J Periodontol 1980 Oct;51(10):553-562. 
30. Friskopp J. Ultrastructure of nondecalcified supragingival and subgingival calculus. J Periodontol 1983 Sep;54(9): 542-550.

31. Shirato M, Kamishikiryo K, Itoh A, Kado H, Maeda $\mathrm{Y}$, Sekiguchi T, Fukui K, Takezawa T. Observations of the surface of dental calculus using scanning electron microscopy. J Nihon Univ School Dent 1981;23:179-187.

32. Patters MR, Landesberg RL, Johansson LA, Trummcl CL, Robertson PB. Bacteroides gingivalis antigens and bone resorbing activity in root surface fractions of periodontally involved teeth. J Periodontal Res1982 Mar;17(2):122-130.

33. Soben Peter. Text book of public health dentistry. 5th ed. Chapter 4. p. 126.

34. Kamath DG, Nayak SU. Detection, removal and prevention of calculus: Literature review. Saudi Dent J 2014 Jan;26(1): 7-13.

35. Archana V. Calculus detection technologies: Where do we stand now? J Med Life 2014;7(Spec Issue 2):18-23. 